\title{
A Conceptual Design of a Comet Explorer Performing both Penetrating and Surface Roving Missions
}

\author{
Yuchen Bai* \\ University of Michigan, Ann Arbor, Michigan, United States \\ Robert Farquhar ${ }^{\dagger}$ \\ KinetX Inc., Washington D.C., District of Columbia, United States \\ Hongcheng $\mathrm{Xu}^{\ddagger}$ \\ Beijing University of Aeronautics and Astronautics, Beijing, P.R.China \\ Xilun Ding $\$$ \\ Beijing University of Aeronautics and Astronautics, Beijing, P.R.China
}

\begin{abstract}
Exploration for comet and asteroid holds a critical position to study the origin of the earth and the solar system. Due to the low gravity situation, it is difficult to send a rover onto the surface of those celestial bodies. This paper gives a preliminary design for penetrating and surface roving system for a proposed comet exploration mission. The system is constituted by two parts: a Boost Vehicle with a surface robotic rover contained, and a Penetration Vehicle with deployable structure. The assembly will be carried by the main spacecraft to execute a fly-by maneuver towards the target comet. System compositions, possible payloads, feasibility trade-offs and initial analyses are also presented in the paper with flowcharts and simulations. The paper finishes with the scenario of a typical task process for the assembly.
\end{abstract}

\section{Introduction}

Comet Exploration is playing an increasing important part in the area of deep space mission from last century, a number of explorers had been launched since 1978. The first success came on September 11th in 1985, when ISEE-3 passed through the tail of comet Giacobini-Zinner [1]. A real climax of comet exploration was in 1986: from March 6th to March 14th, five spacecraft were sent into the universe by USA, USSR, Europe and Japan for a same target - the comet Halley [2]. The most exciting breakthrough in comet detection was at 5:52 UTC on July 4, 2005, the impactor of the Deep Impact space probe collided with the nucleus of comet Temple 1 and excavated debris from the interior of it [3] [4]. Although we have made great achievements on comet detection, there is still no explorer soft landing on the surface of the comet. Because of the extremely weak gravity field of the comet, it would not be easy to keep the surface exploration robot onto the ground, or it may bounce back to the space.

This paper provides a conceptual design scheme of independent penetrating and landing system for the mission, it is an additional module for a proposed multi-comet mission in 2018. Our autonomous spacecraft is constituted by a Boost Vehicle (BV) with a "spider" surface robotic rover $\left(S R^{2}\right)$ contained, and a Penetration Vehicle (PV) with light deployable "cobweb" structure. The assembly will be carried by the main spacecraft to execute a fly-by maneuver towards the target comet and released. After an autonomous flight phase, the

\footnotetext{
*Research Assistant, Department of Aerospace Engineering, baiyc@umich.edu

$\dagger$ Executive, Space Exploration, robert.farquhar@kinetx.com

$\ddagger$ Research Assistant, Robotics Institute

$\S$ Professor, Robotics Institute, xlding@buaa.edu.cn
} 
cobweb structure will anchor on the comet surface by the penetrator and fixing stakes, and the spider rover will be released by the booster onto the cobweb and crawl through it for surface exploration. The system structure of the assembly, a preliminary CATIA model, primary goals for each component and detailed design scheme with control methods and structures were given and analyzed to realize the mission requirements. Possible payloads for the main spacecraft and the assembly were also introduced in the paper. The paper finishes with the scenario of a typical task process for the assembly, including the deployment of the cobweb and the landing procedure of the surface rover.

\section{System Design}

For the proposed comet mission, a preliminary design of an assembly of penetrator and booster is given, a sketch of the assembly is shown in Figure 1.

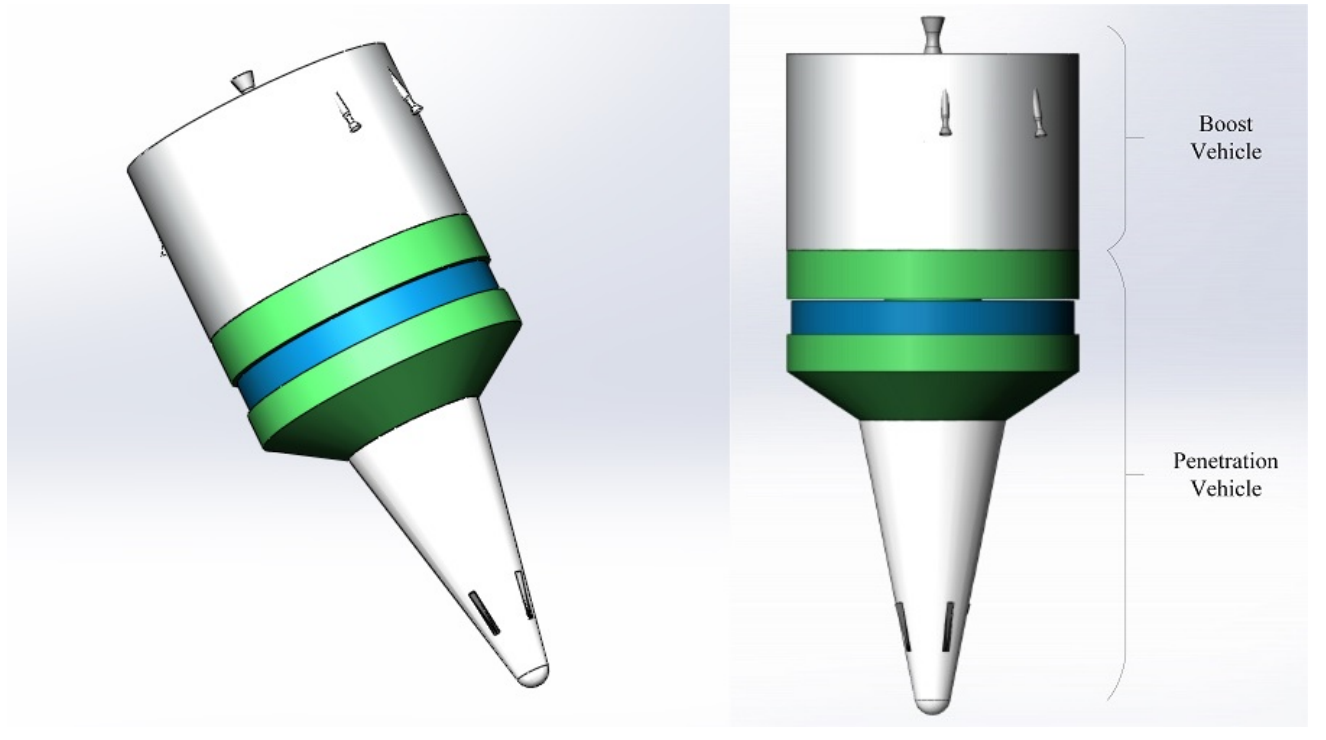

Figure 1. (Left) a sketch of the assembly; (Right) two parts of the assembly.

The initial intent of the design is to integrate penetration system and surface exploration system in a single spacecraft which could be an optional payload for the comet flyby mission. The assembly contains all the hardware which is necessary for comet surface exploration, and only maintains information link with the main spacecraft using data chain. The system is provided with a high level of intelligence and automation to carry out an independent detection task.

The assembly has two parts: the Boost Vehicle (BV) and the Penetration Vehicle (PV). The BV has the capability to contain the Surface Robotic Rover $\left(S R^{2}\right)$ for surface roving program, and the PV could install the deployable cobweb structure as supporting facility for the rover to climb on. Figure 2 shows the system structure of the assembly: both BV and PV have independent electric energy sources; the liquidpropellant rocket system and GNC module are designed on the BV, the attitude adjustment is executed by both liquid-propellant rocket system and reaction wheels on the PV. The PV's final approach to the target is an unpowered glide along the route before BV's separation, and the attitude of PV could be slightly adjusted by reaction wheels. The facilities circled by dotted line are landing payload, they should be removed together in case of a more basic task with less budget.

\section{A. Boost Vehicle}

The Boost Vehicle (BV) is designed to:

1. Accelerate the Penetration Vehicle (PV) to an appropriate velocity to wedge into the target surface successfully;

2. Relay data and guidance information between PV and main spacecraft after PV's separation; 


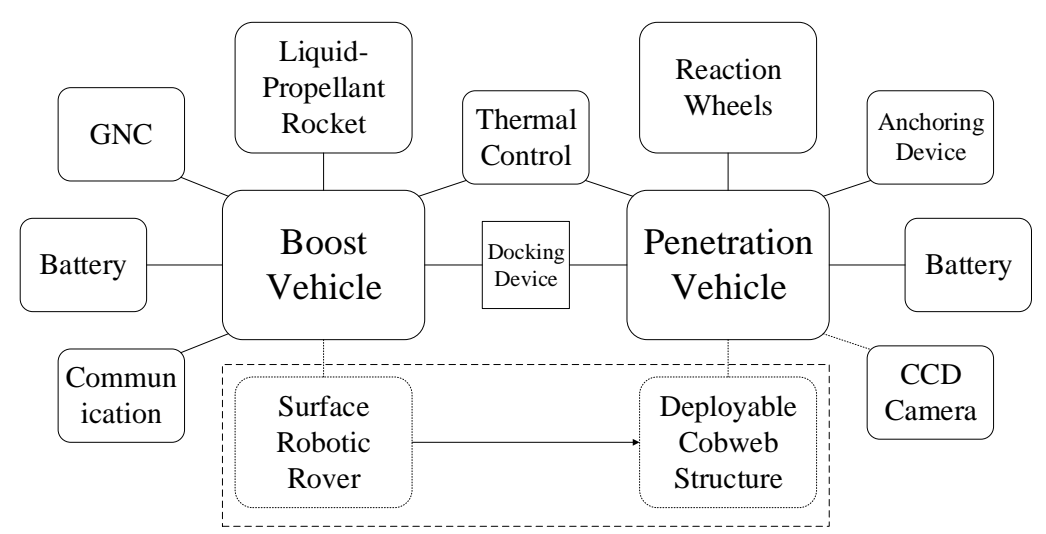

Figure 2. System structure of the assembly.

3. Contain the Surface Robotic Rover $\left(S R^{2}\right)$ during the accelerating and braking phases of the BV;

4. Release $S R^{2}$ to the target area covered by the cobweb structure.

To achieve the goals above, the Boost Vehicle is planned to use a liquid-propellant rocket engine system which can easily ignition multiple times to provide indispensable speed and attitude control. The choice of fuel and oxidizer should meet the demand of the capability of multiple times re-ignitions. Thus, some possible options of the fuel could be mono-methyl hydrazine $\left(\mathrm{CH}_{6} \mathrm{~N}_{2}\right)$ or dimethyl hydrazine $\left(\mathrm{C}_{2} \mathrm{H}_{8} \mathrm{~N}_{2}\right)$, and the oxidizer should be nitrogen tetroxide $\left(\mathrm{N}_{2} \mathrm{O}_{4}\right)$. There is also a pressurant tank containing Helium to supply gas for the engine system.

One Main Axial Engine (MAE) and 6 Attitude Control Engines (ACE) are built in, including 2 engines for rolling control and 4 engines for pitching and yawing. All of the attitude control engines are designed on the edge of the BV which is shown in Figure 3, they are all identified as their polar angles on the x-y plate. The ACEs for pitching and yawing are distributed as two groups close to $0^{\circ}$ and $180^{\circ}$ direction, it will be easier for the layout of fuel and oxide pipelines.

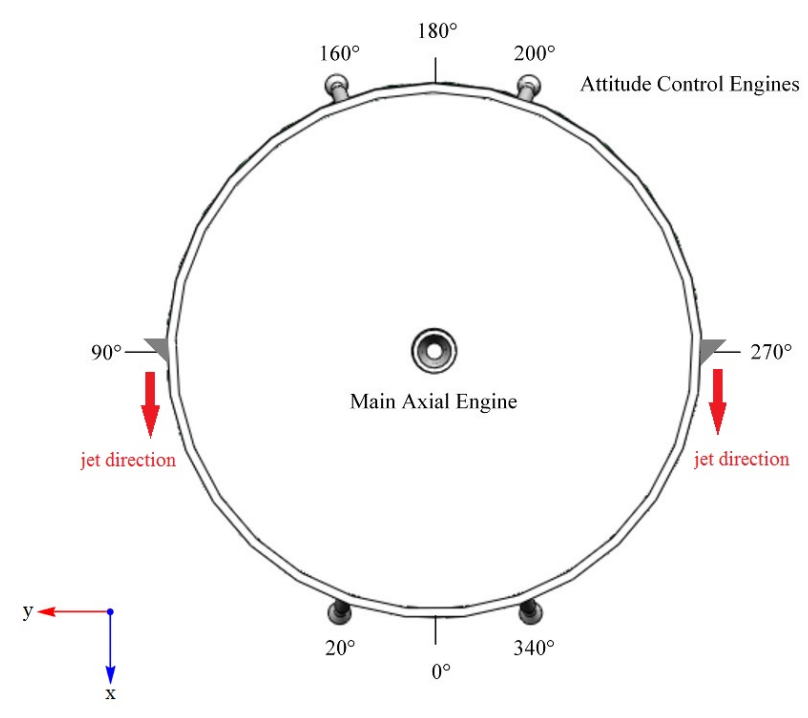

Figure 3. Propellant and attitude control engines on the Boost Vehicle (seen through the positive z-axial direction of the $\mathrm{BV})$. 
The pitching and yawing engines can provide thrust approximately through the z negative direction with a small angle against the axis, by using a short time burn. We can cleverly arrange the combustion sequence of the ACEs to correct the assembly's attitude. In Table 1, a form shows the burn procedures of the six ACEs for different types of maneuver, the direction of ACE is same as Figure 3.

\begin{tabular}{c|c|c|c|c|c|c}
\hline & ACE: $20^{\circ}$ & ACE: $90^{\circ}$ & ACE: $160^{\circ}$ & ACE: $200^{\circ}$ & ACE: $270^{\circ}$ & ACE: $340^{\circ}$ \\
\hline $\begin{array}{c}\mathrm{x}+ \\
(\text { Yaw })\end{array}$ & 2 & $/$ & 2 & 1 & $/$ & 1 \\
\hline $\begin{array}{c}\mathrm{x}- \\
(\text { Yaw })\end{array}$ & 1 & $/$ & 1 & 2 & $/$ & 2 \\
\hline $\begin{array}{c}\mathrm{y}+ \\
(\text { Pitch })\end{array}$ & 1 & $/$ & 2 & 2 & $/$ & 1 \\
\hline $\begin{array}{c}\mathrm{y}- \\
(\text { Pitch })\end{array}$ & 2 & $/$ & 1 & 1 & $/$ & 2 \\
\hline $\begin{array}{c}\text { z+ } \\
(\text { Roll })\end{array}$ & $/$ & 1 & $/$ & $/$ & 2 & $/$ \\
\hline $\begin{array}{c}\text { z- } \\
(\text { Roll })\end{array}$ & $/$ & 2 & $/$ & $/$ & 1 & $/$ \\
\hline $\begin{array}{c}\text { BV } \\
\text { BRAKE }\end{array}$ & 1 & $/$ & 1 & 1 & $/$ & 1 \\
\hline
\end{tabular}

Table 1. Combustion sequences for different maneuvers.

In Table 1, the first column shows the axis which the assembly maneuvers around, the first row represents each attitude control engine on the BV. The number 1 and 2 show the ignition order of the ACEs to execute a maneuver in the first column, the engines with same number ignite and shut down simultaneously, and "/" means the ACE do not need to ignite in the corresponding procedure. One important thing is: a pulse burn of one engine group will give the probe an extra angular momentum, it should be neutralized by an angular momentum with equal intensity and opposite direction. That is why all burns in Table 1 should be symmetric.

Figure 4 is a proposed standard re-ignitable liquid-propellant rocket system design for the Boost Vehicle. A pressure regulator is devised in the main gas supply line (black line in Figure 4) to adjust the input of Helium, a slam suppressor, an isolation valve and a relief valve is also built in or on the main gas pipeline. The gas line separates into two, and they are respectively guided to the fuel tank and oxidant tank to provide continuous pressure on the liquid components. There are isolation valves in main fuel line (red line in Figure 4 before filters), main oxidant line (blue line in Figure 4 before filters) and each branch of gas lines for safety consideration. Two pressure transducers are designed as monitor and feedback devices in both fuel and oxidant main lines. Considering that the main axial engine has a fuel flow larger than any attitude control engine, it has independent pipes for fuel and oxidant supply, and all ACEs share another branch of the main fuel and oxidant line.

An integrated Guidance, navigation and control (GNC) system is designed in the Boost Vehicle to realize the related objectives, the sketch is shown in Figure 5. The BV receives directives of the main spacecraft after being released from it. The navigation camera system located on the penetrator continuously captures and transmits image signals to the on-board computer through the trajectory filter. The GNC system uses multiple sensors to detect the status of the spacecraft in real-time, including star sensor and Inertial Measurement Unit (IMU). The on-board computer has processors for attitude and trajectory control, by using attitude guidance algorithm and trajectory guidance law planted in. It processes data and gives instructions to guide the assembly towards the target area on the comet in full automation.

Communication system should realize the real-time downlink of the main spacecraft instruction, the timing uplink of measurement data and contact between surface rover and the main spacecraft. It makes use of the current vulgate $\mathrm{X}$ band radar transceiver unit and uplink-downlink communicator. The data accords with the international CCSDS standard. The Boost Vehicle gets electric energy supply from an independent 


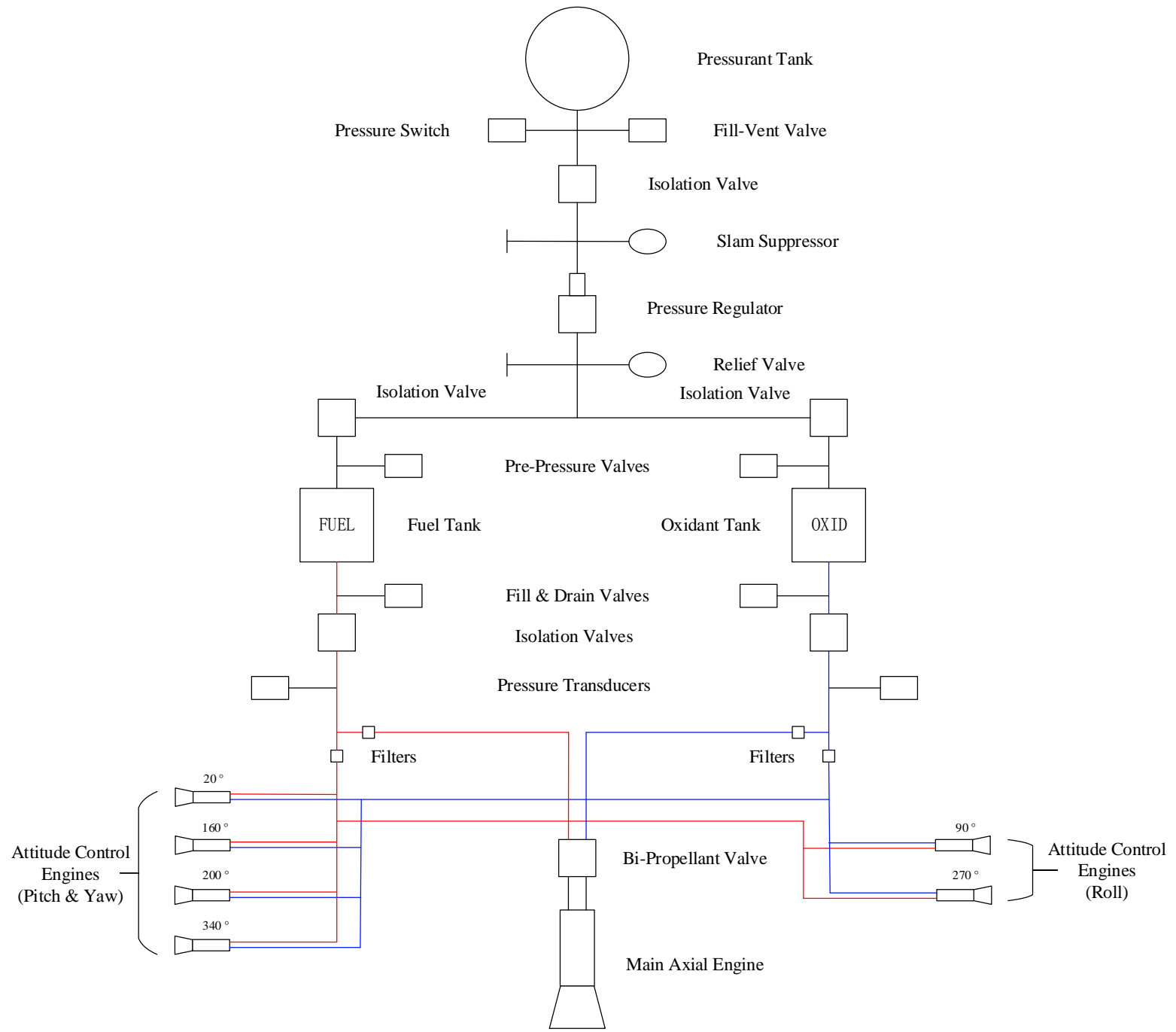

Figure 4. Design of liquid-propellant rocket engine system on the Boost Vehicle.

storage battery group on board, an extra solar panel is optional for a larger and more functional project to provide continuous energy on charging the battery group. A basic thermal control system is also planned on the assembly to keep the equipment in working temperature range. Next to the docking device with Penetration Vehicle, there is a specific compartment for the Surface Robotic Rover $\left(S R^{2}\right)$.

\section{B. Penetration Vehicle}

The penetration vehicle (PV) is designed to:

1. Contain the cobweb structure and deploy it after separation from the Boost Vehicle;

2. Execute an unpowered final approach to the target and upload data to the Boost Vehicle;

3. Penetrate the comet surface and anchor on it to be the infrastructure of the Surface Robotic Rover.

Figure 6 shows the side view of the Penetration Vehicle, the preliminary geometric appearance of PV includes a cone, a cone frustum and a cylinder. The cobweb structure is designed in the cylinder part, an annular sheath (the blue part in Figure 6) which could be open from the front protects the flexible structure. The right side picture in Figure 6 shows the open status of the sheath, and the cobweb is deployed. 


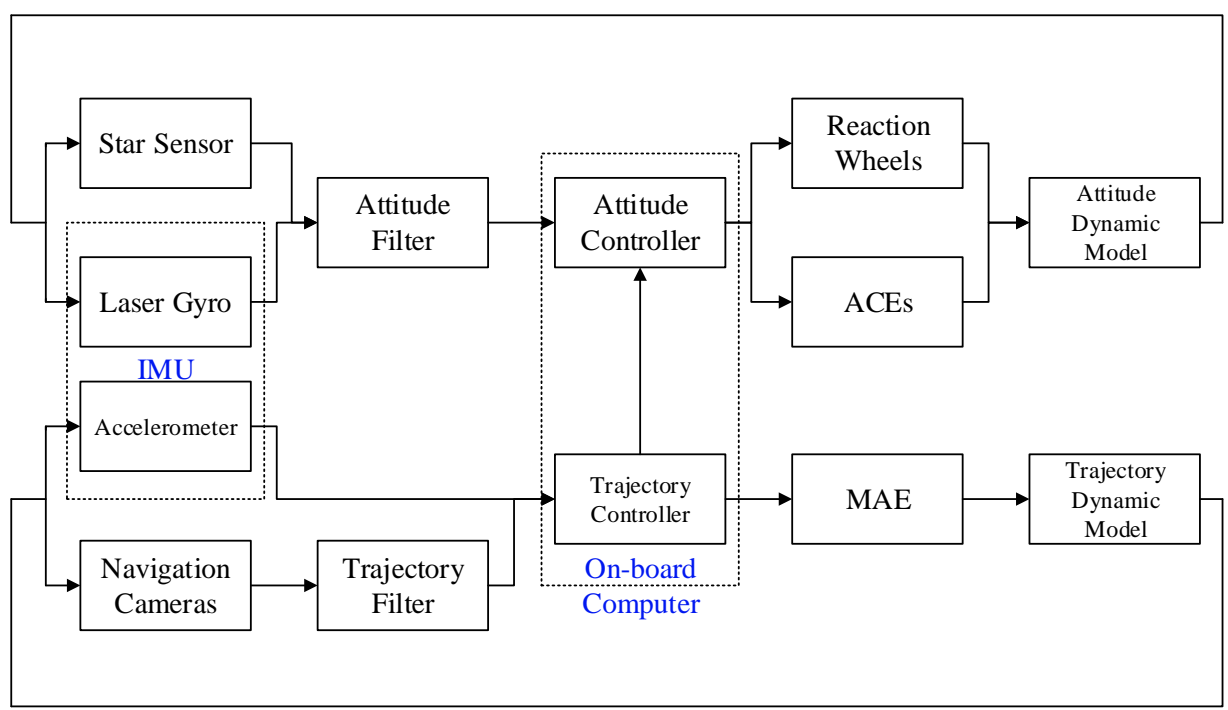

Figure 5. Sketch of the GNC system structure.

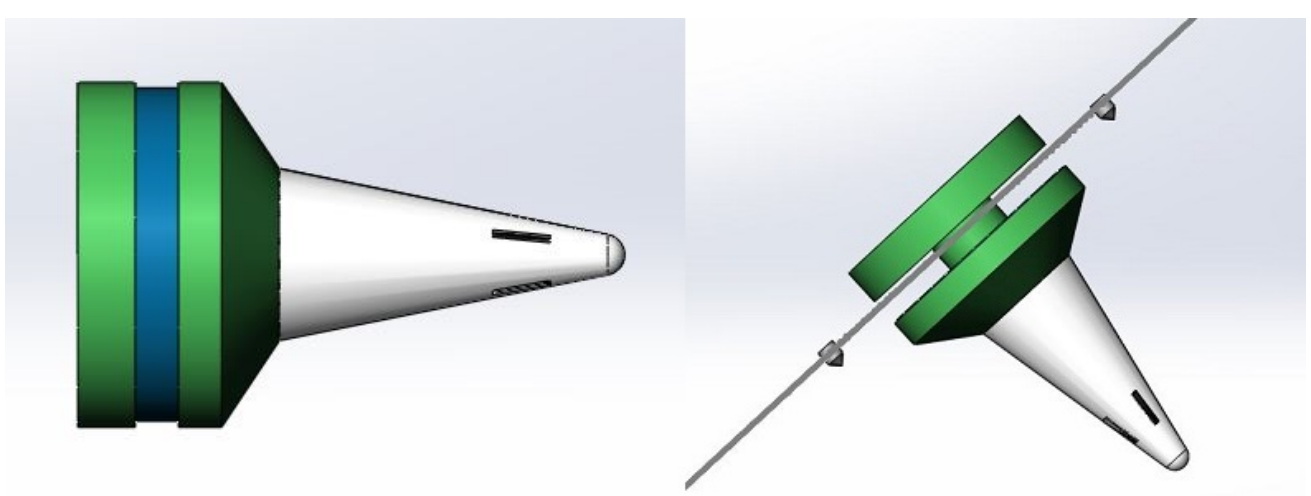

Figure 6. Side view of Penetration Vehicle (left side shows the cobweb not deployed and the anchor retracted).

The Deployable Cobweb Structure is the antecedent facility of the Surface Robotic Rover. Due to the extremely low gravity of the comet, the traditional wheeled or crawler-type detector is almost impossible to gain enough static friction to run. Also, the escape velocity is very small and makes the hopping or legged robot difficult to work, it can easily hop out of the comet gravity sphere.

Theoretically, there is no way to send a surface explorer onto a comet without any junction point with it, and that is why the deployable cobweb structure is designed for the landing mission.

Figure 7 is a preliminary sketch of the Cobweb Structure, a concentric regular hexagonal net. The maximum side length of hexagon is planned as approximate $20 \mathrm{~m}$, which gives the surface rover about $1000 \mathrm{~m}^{2}$ area to detect. On each vertex of the largest hexagon, there is a stake attached to fix the cobweb onto the comet surface (shown in the corner of Figure 7). When the deployment instruction is received, the release mechanism ejects all the 6 stakes centrifugally, symmetrically and simultaneously, the cobweb plane is perpendicular to the velocity vector of the Penetration Vehicle. Based on the release methods, the complete deployment process will cause no major attitude perturbation on the system, the minor distribution caused by the full extension of cobweb and the motion of stakes can be corrected by reaction wheel system on-board.

When the Penetration Vehicle hits the ground, its cone shape front will pierce through the comet surface, and the cone frustum part will stuck outside to make sure the cobweb system and communication antenna 


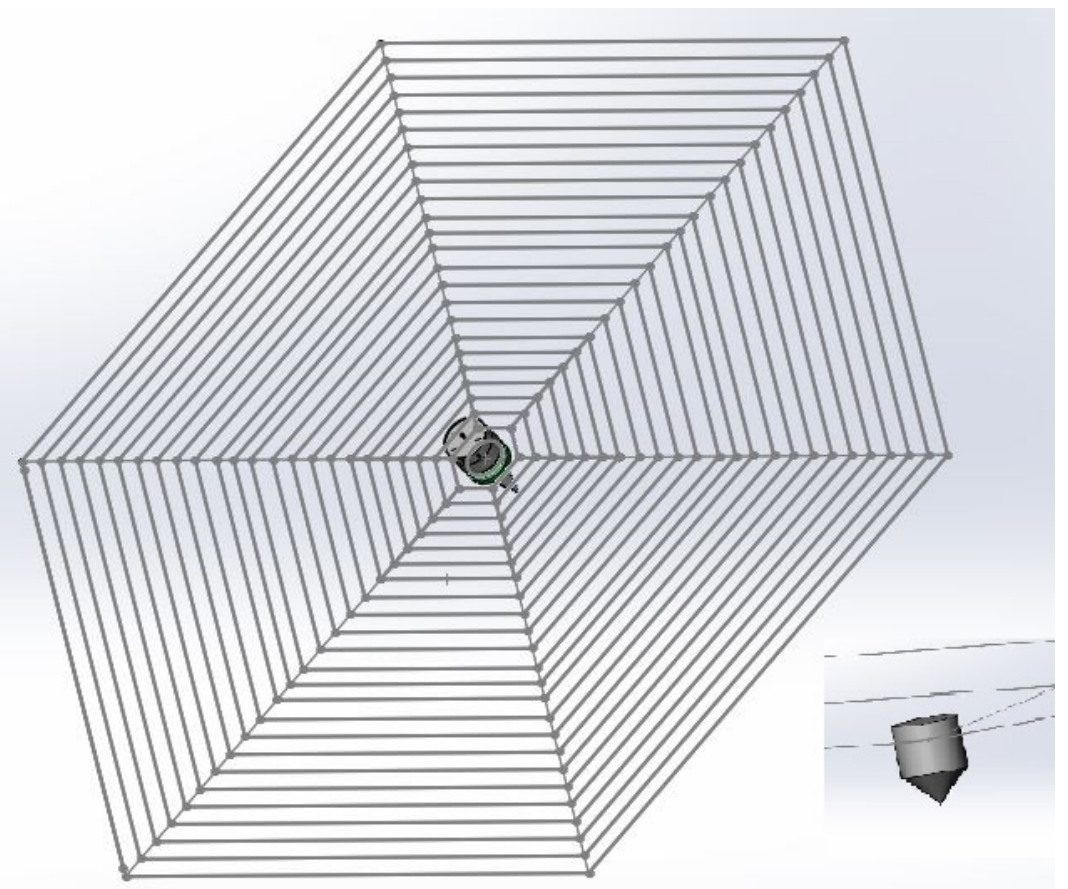

Figure 7. Sketch of the Cobweb Structure and the fixing stake.

in the cylinder rear part are above the ground. The anchoring device in the very front of the Penetration Vehicle will pop out automatically, creating barbs to ensure a strong junction, Figure 8 shows details of anchoring device.

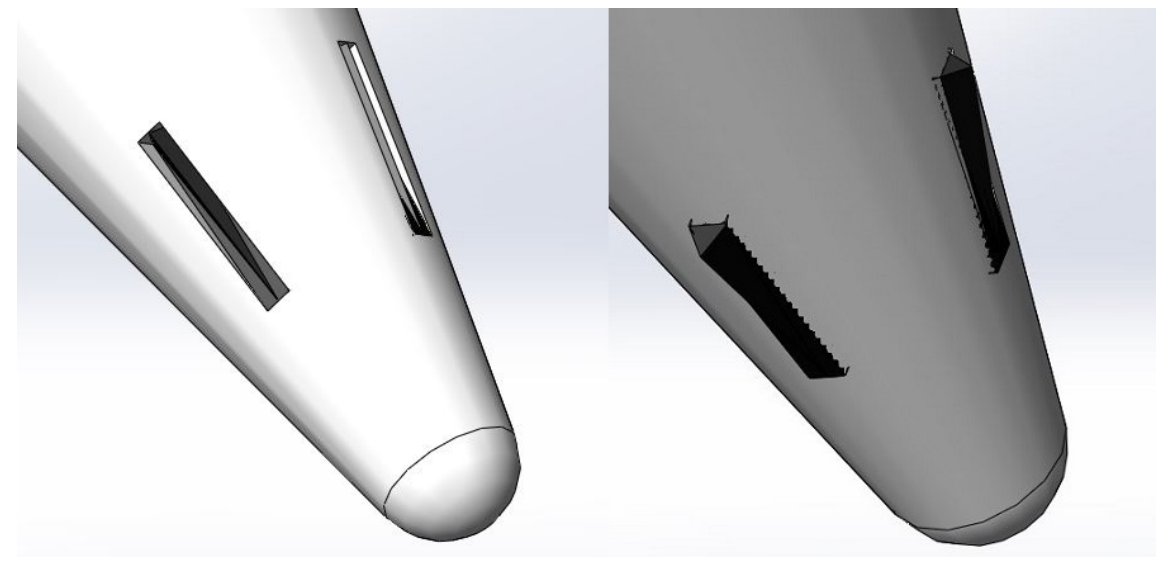

Figure 8. Anchoring Device (retract on the left picture and open on the right).

\section{Surface Robotic Rover}

The Surface Robotic Rover $\left(S R^{2}\right)$ is designed to:

1. Release by the Boost Vehicle and land to the cobweb structure fixed on the comet surface;

2. Automatically crawl along the cobweb and explore the area covered by the cobweb;

3. Provide electric power to keep the payloads working intermittently, return data to the main spacecraft; 
4. Sample the comet and launch a return module to the main spacecraft (optional).

It is carried in the compartment of Boost Vehicle and released after the Penetration Vehicle and cobweb structure successfully fix on the comet. There is no propellant system equipped on the rover so the initial descending velocity is given by the release mechanism. We provide two different configurations for the Surface Robotic Rover: a duel-arm space robot and a radial symmetrical six-legged robot [5] (both in Figure 9). The robotic rover has the capability to capture the web and claw on it, keeping at least one escapement on the arms or legs fixed with the cobweb in movement and static state.
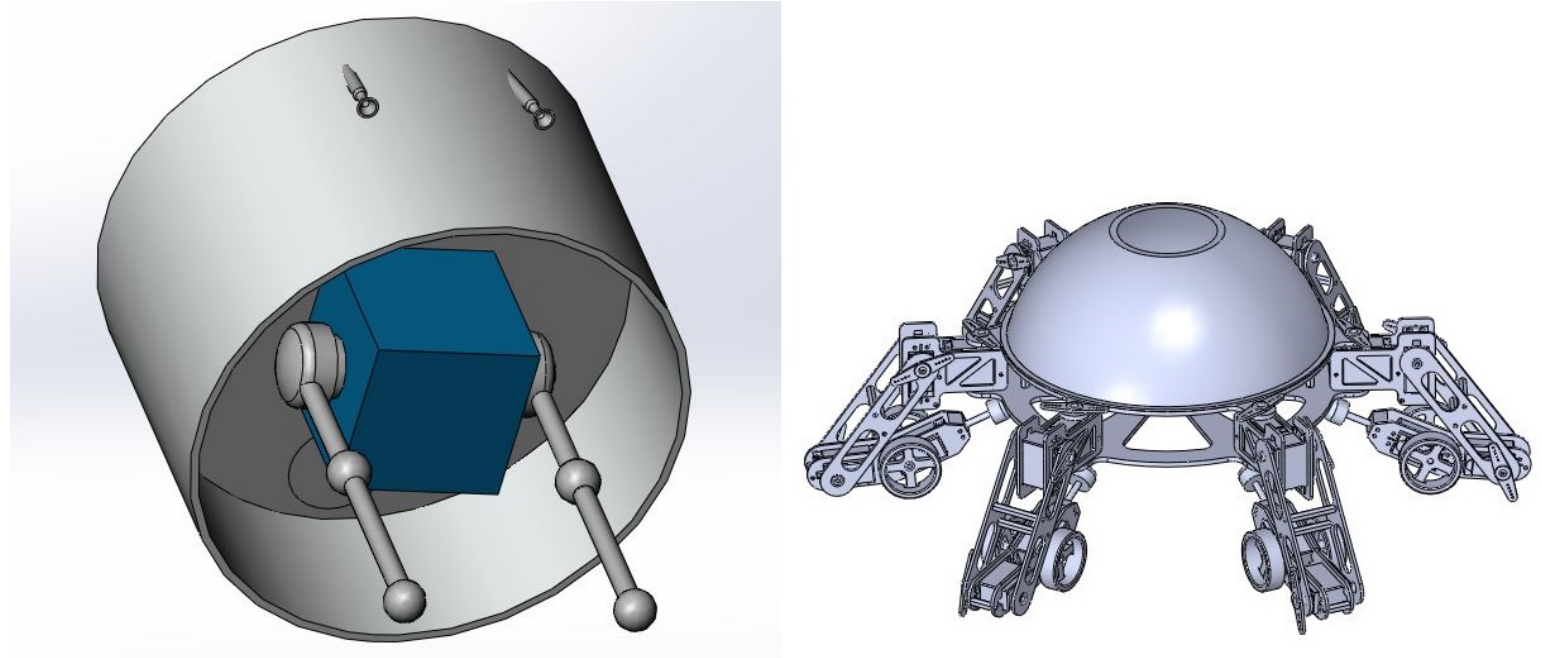

Figure 9. $\mathrm{SR}^{2}$ compartment in the Boost Vehicle and optional schemes of $\mathrm{SR}^{2}$.

The Surface Robotic Rover can automatically explore the area covered by cobweb, by crawling around through the deployed structure. Based on the payloads, the rover can do scientific experiments and sampling, the on-board microscopic camera can get a closer view of the comet surface and transmit the data to the main spacecraft. To sustain the long time detecting ability, the rover is covered by the solar cell "flakes" to convert solar power into electric energy and storage in the battery units. Because of the rotation and revolution of the comet, the sunlight supply might be intermittently, and the $S R^{2}$ has a self-protection program to switch into hibernation during a low battery or extreme temperature status. The $S R^{2}$ also retain the potential to equip an integrated sampling and returning system for an extended mission.

\section{Typical Mission Process}

We introduce a multi-comet flyby mission opportunity in 2018 proposed by Dr. Robert Farquhar [6]. He and his collaborators designed a 1.4 year Earth-return trajectory to Comet Wirtanen, then to SW3-C in 2022, Figure 10 is the Earth- return trajectory with Wirtanen and the trajectory to SW3-C. The assembly of Boost Vehicle and Penetration Vehicle could be loaded by the main spacecraft of this proposed mission to penetrate and land on Wirtanen.

The explorer is suggested to launch on March 18th, 2018. The essential payloads of the main spacecraft include an image acquisition system, an infrared speculator and a comet dust analyzer. During the cruising phase, the penetrator assembly will keep in dormant state until the main spacecraft closing the target comet. When Wirtanen is approximately 7 days away, the flyby spacecraft will execute a series of maneuver to target the comet and aim the assembly directly to it. Then the assembly separates from the main spacecraft and ignites the liquid-propellant rocket engine to accelerate, the auto-navigation system adjusts the trajectory to the target area, multiple correction maneuvers will be implemented by the Boost Vehicle to ensure the Penetration Vehicle in the exact speed and attitude. The separation of BV and PV is executed by the release of docking device with a braking maneuver by the Boost Vehicle at the same time, the Penetration Vehicle glides to the target area on the comet without propellant. Minutes before separation when the Penetration Vehicle is clear to the plume influence of the Boost Vehicle, the cobweb structure on PV will 


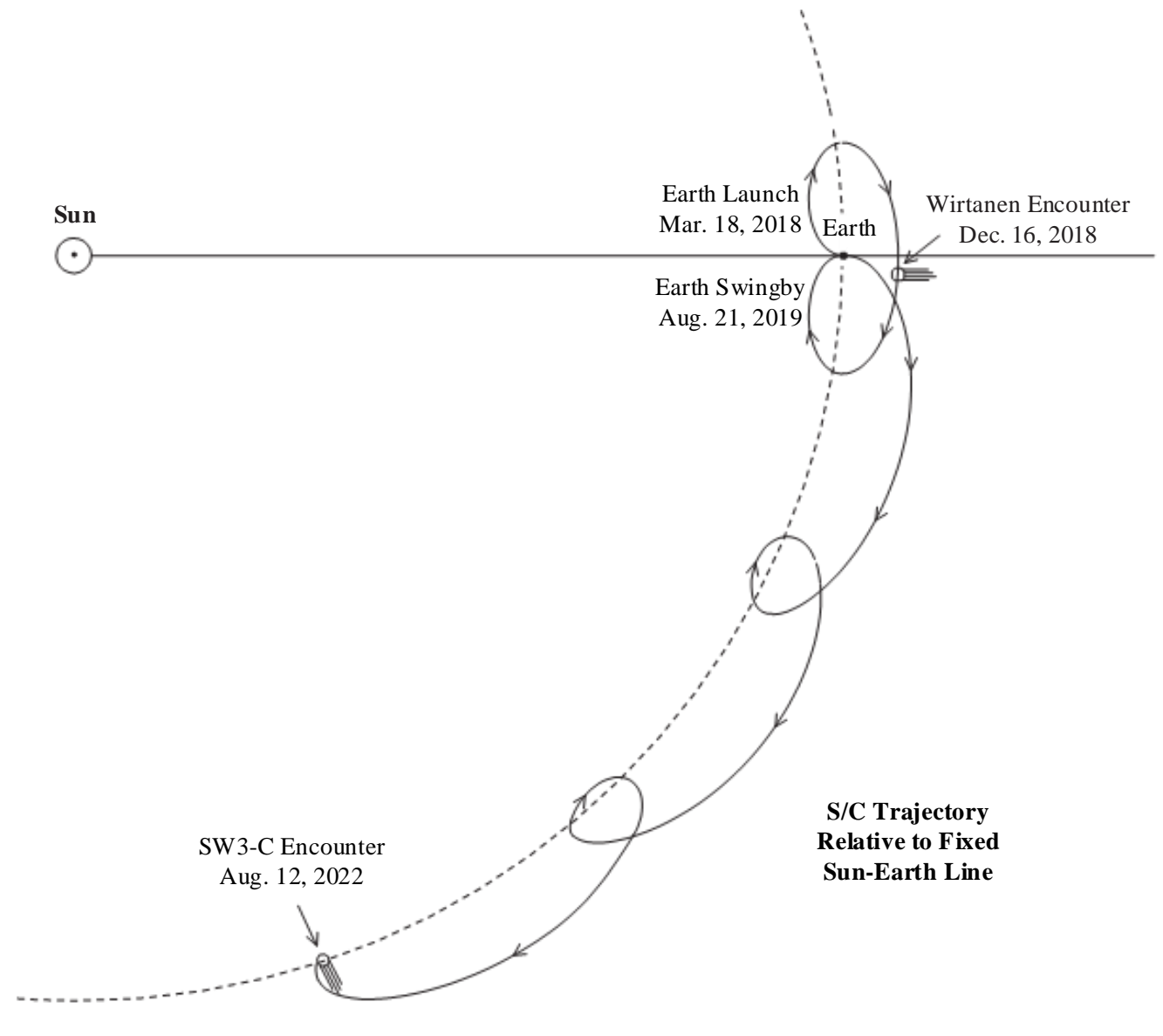

Figure 10. Trajectory to Wirtanen and SW3-C [6].

be deployed: 6 fixing stakes are popped out by the release mechanism simultaneously and perpendicularly to the velocity of PV, driving the flexible cobweb to the fully deployed position. The reaction wheels will correct the disturbance caused by the deployment and keep the Penetration Vehicle in a proper attitude for piercing the comet surface, a wrong angle against the surface will lead to a ricochet. The navigation CCD cameras will visually capture the comet and upload image data to the Earth control center with relays from Boost Vehicle and main spacecraft till the Penetration Vehicle piercing the ground.

When the Penetration Vehicle hit the comet, the front part will penetrate the surface of target area and stuck on the ground, triggering the barbs to anchor PV. Milliseconds later the 6 fixing stakes also touch down, similar to the penetrator, the impact will induce the micro-harpoon inside each stake shooting into the surface. The cobweb structure is pinned on the target area permanently by the Penetration Vehicle and fixing stakes and ready for the landing of Surface Robotic Rover. During the final approach phase and impact phase of the assembly, the main spacecraft will execute a deflection maneuver to realize a flyby of Wirtanen with a closest approach distance about $500 \mathrm{~km}$. The image acquisition system and the dust analyzer on-board will collect about the collision and the dust erupted. After confirming the successful fixation of the cobweb structure, the Boost Vehicle will automatically adjust the attitude and trajectory to aim on the cobweb and release the Surface Robotic Rover. The decent phase of $S R^{2}$ is also unpowered, the initial velocity is given by the Boost Vehicle. The arms/legs of the rover will grasp the cobweb and lock on it to land on the surface of the comet without rebound. The rover can crawl over the web to finish the exploration mission, and return scientific data to the main spacecraft. 


\section{Conclusion and Future Work}

In this paper we made a preliminary system design of an assembly of penetrator and booster for a proposed multi-comet flyby mission. The system structure of the assembly, the primary goals for each component and detailed design scheme were given to realize the mission requirements. We also discussed a typical mission process and possible payloads for the main spacecraft and the assembly. The concept of this assembly can also be used on a mission to other low gravity celestial bodies, such as objects in main asteroid belt and Kuiper belt.

The future research will focus on the specific design and optimization of the vehicles' assembly ,by using Multidisciplinary Design Optimization (MDO) methods [7]. To achieve a better understanding of the mission, additional studies about the speed condition, shape of the penetrator, mass control and overall cost accounting must be extended to. For instance, Figure 11 shows an eXtended Design Structure Matrix (XDSM) of the physical problems, coupling in the level of discipline. The coming work is to decouple the XDSM, using optimization controller to process the conflict of demands and constraints among different subsystems. Also, detailed design and simulation of the Surface Robotic Rover and the deployable cobweb will continue, necessary improvements will be implemented for some celestial bodies with high rotation speed.

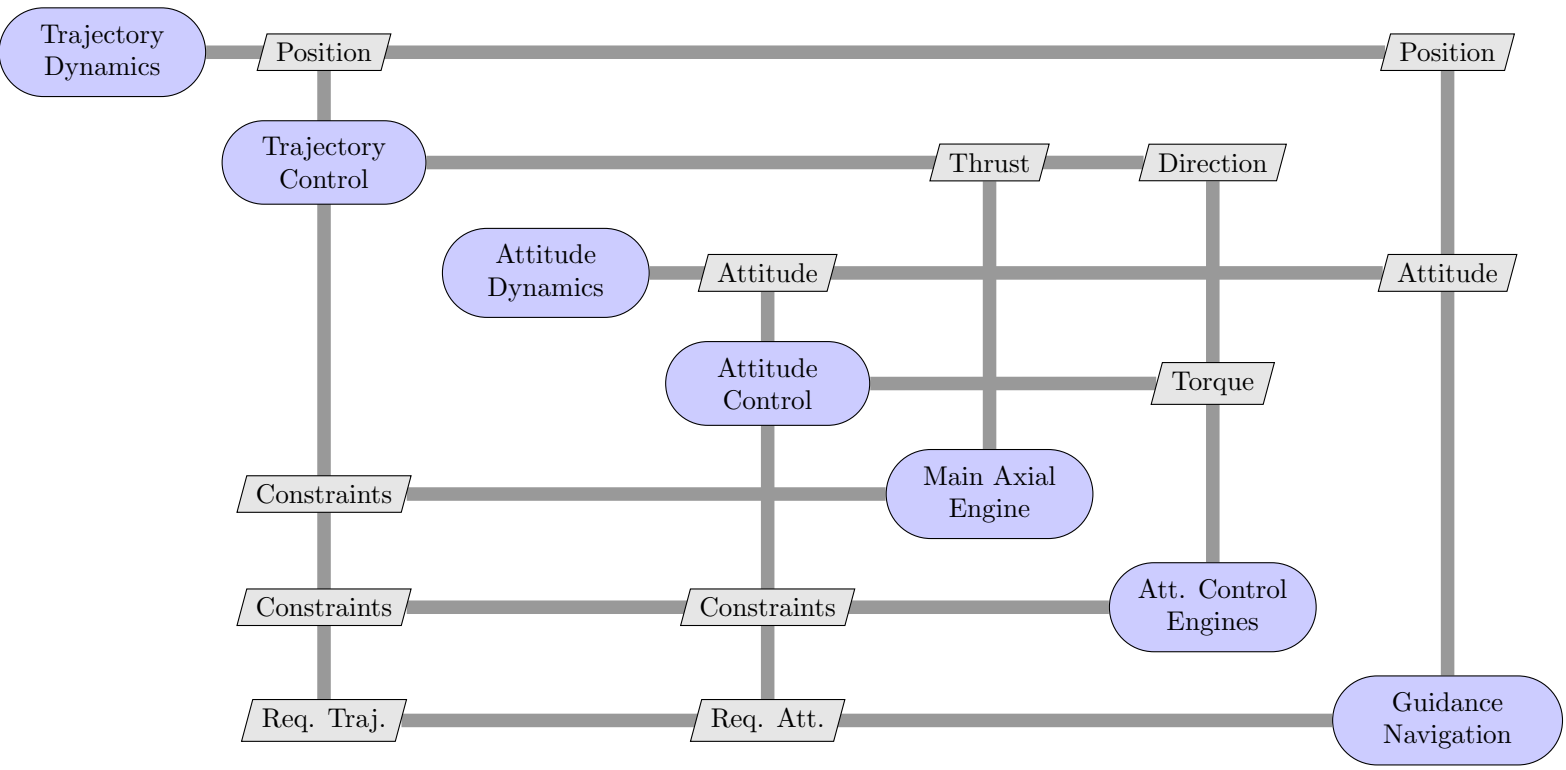

Figure 11. The eXtended Design Structure Matrix diagrams for the true problem.

\section{Acknowledgments}

The authors would like to thank Prof. Joaquim R. R. A. Martins for his development in Multidisciplinary Design Optimization, and his XDSM compiler as well.

\section{References}

\footnotetext{
${ }^{1}$ Murdin, P. "International Cometary Explorer (ICE)." Encyclopedia of Astronomy and Astrophysics 1 (2000), pp. 4650.

${ }^{2}$ Keller, H. U., Kramm, R., \& Thomas, N. "Surface features on the nucleus of comet Halley." Nature 331 (1988), pp.

${ }^{3}$ Veverka, J., et al. "Return to Comet Tempel 1: Overview of Stardust-NExT results." Icarus 222.2 (2013), pp. 424-435.

${ }^{4}$ A'Hearn, Michael F., et al. "Deep impact: excavating comet tempel 1." Science 310.5746 (2005), pp. 258-264.

${ }^{5}$ Wang, Zhiying, et al. "Mobility analysis of the typical gait of a radial symmetrical six-legged robot." Mechatronics 21.7 (2011), pp. 1133-1146.
} $227-231$ 
${ }^{6}$ Farquhar, R., et.al. "A Unique Multi-Comet Mission Opportunity For China In 2018." 64th International Astronautics Congress, A3.4.12.

${ }^{7}$ Martins, Joaquim RRA, and Andrew B. Lambe. "Multidisciplinary design optimization: a survey of architectures." AIAA journal 51.9 (2013): 2049-2075.

11 of 11 